\title{
Effects of inhomogeneity on surface waves in anisotropic media
}

\author{
PRANABES KANTI PAL ${ }^{1}$ and D ACHARYA ${ }^{2}$ \\ ${ }^{1}$ Indrapuri, Ichapur 743144 , India \\ ${ }^{2}$ Department of Mathematics, Mahadevananda College, Barrackpur 743 101, \\ India
}

MS received 17 July 1997; revised 11 May 1998

\begin{abstract}
This paper investigates the effects of anisotropy and inhomogeneity on surface waves in elastic media. Exponential variation in properties are assumed for the elastic parameters and material density. The classical equations of motion for propagation of waves in an inhomogeneous transversely isotropic elastic solid are deduced. The equations of motion for surface waves are derived and general surface waves are investigated. This general theory is then utilized to investigate Rayleigh, Love and Stoneley waves. Results obtained in the above cases reduce to the corresponding well-known classical results when inhomogeneity and anisotropy are not present. It is seen that inhomogeneity has significant effects on dispersion characteristics. Numerical calculations are included for Love waves and some conclusions have been drawn from the above calculations.
\end{abstract}

Keywords. Exponential inhomogeneity; transversely isotropic; surface waves; Rayleigh waves; Love waves; Stoneley waves; wave velocity equation.

\section{Introduction}

It is known that surface waves in a homogeneous isotropic elastic solid medium play an important role in the study of earthquakes, seismology, geophysics and geodynamics. The theory of surface waves has been widely investigated by Rayleigh (1885), Stoneley (1924), Ewing et al (1957), Hunter (1960), Bland (1960), Biot (1965), Flugge (1967), and Jeffreys (1970). The effects of the anisotropic character of the medium on surface waves have been studied to a limited extent. The problem of wave-propagation in an inhomogeneous medium also plays a role, because any realistic model of the earth must take into account continuous changes in the vertical direction of the elastic properties of the material. Some problems of wave propagation, in which viscosity, rigidity and density are functions of depth, have been studied by several authors (Dutta 1963; Bhattacharya 1969; Chattopadhyay 1975). In this paper, we consider the problem of surface waves 
when inhomogeneity and anisotropy occur simultaneously. We assume the medium to be transversely isotropic which is a particular case of anisotropy. Following the papers of Dey \& Sengupta (1978) and Das et al (1992), we assume the inhomogeneities of the lower medium $\left(M_{1}\right)$ and the upper medium $\left(M_{2}\right)$ to be respectively of the form:

$$
\begin{aligned}
& A=A_{1} e^{\nu z}, \quad N=N_{1} e^{\nu z}, \quad F=F_{1} e^{\nu z}, \\
& C=C_{1} e^{\nu z}, \quad L=L_{1} e^{\nu z}, \quad \rho=\rho_{1} e^{\nu z}, \\
& A^{\prime}=A_{1}^{\prime} e^{v^{\prime} z}, \quad N^{\prime}=N_{1}^{\prime} e^{v^{\prime} z}, \quad F^{\prime}=F_{1}^{\prime} e^{v^{\prime} z}, \\
& C^{\prime}=C_{1}^{\prime} e^{v^{\prime} z}, \quad L^{\prime}=L_{1}^{\prime} e^{v^{\prime} z}, \quad \rho^{\prime}=\rho_{1}^{\prime} e^{v^{\prime} z},
\end{aligned}
$$

where $A_{1}, N_{1}, F_{1}, C_{1}, L_{1}, \rho_{1}$ and their primes are constants for an anisotropic medium. In the case of Love waves, the inhomogeneities in two media are assumed to be of the form:

$$
\begin{gathered}
N=N_{1} e^{\nu z}, \quad L=L_{1} e^{\nu z}, \quad \rho=\rho_{1} e^{\nu z}, \quad \text { for } \quad 0 \leq Z \leq \infty, \\
N^{\prime}=N_{1}^{\prime} e^{v^{\prime} z}, \quad L^{\prime}=L_{1}^{\prime} e^{v^{\prime} z}, \quad \rho^{\prime}=\rho_{1}^{\prime} e^{v^{\prime} z}, \quad \text { for } \quad-H \leq Z \leq 0 .
\end{gathered}
$$

We first deduce a set of dynamical equations of motion for a three-dimensional problem with inhomogeneity and transverse isotropy. The general theory of surface waves is then deduced to highlight the effects of anisotropy and inhomogeneity. It is believed that the above type of problem has not so far been considered.

\section{Formulation of the problem and its solution}

Let $M_{1}$ and $M_{2}$ be two nonhomogeneous transversely isotropic semi-infinite elastic solid media, which are in perfectly welded contact along $Z=0, M_{2}$ being above $M_{1}$ (figure 1 ). We consider a system of orthogonal Cartesian axes $O x y z$, the origin $O$ being on the interface $Z=0$ and $O Z$ is normal to the interface vertically downwards.

We consider the possibility of a type of wave along the positive direction of the $x$-axis and largely confined to the neighbourhood of $Z=0$; and the particles in $Y Z$ plane with equal phase. The first assumption asserts that the wave is a surface wave and the second

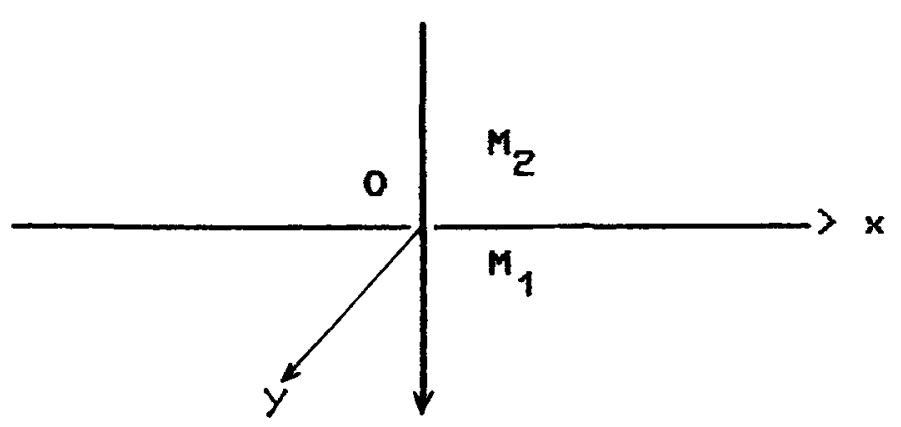

Z

Figure 1. Schematic of the problem. 
assumption leads to zero partial derivatives with respect to $Y$. The equations of motion are

$$
\begin{aligned}
& \frac{\partial \sigma_{11}}{\partial x}+\frac{\partial \sigma_{12}}{\partial y}+\frac{\partial \sigma_{13}}{\partial z}=\rho \frac{\partial^{2} u}{\partial t^{2}}, \\
& \frac{\partial \sigma_{21}}{\partial x}+\frac{\partial \sigma_{22}}{\partial y}+\frac{\partial \sigma_{23}}{\partial z}=\rho \frac{\partial^{2} v}{\partial t^{2}}, \\
& \frac{\partial \sigma_{31}}{\partial x}+\frac{\partial \sigma_{32}}{\partial y}+\frac{\partial \sigma_{33}}{\partial z}=\rho \frac{\partial^{2} w}{\partial t^{2}},
\end{aligned}
$$

where $u, v, w$ are the components of displacement and the stress tensors $\sigma_{i j}(i, j=1,2,3)$ are given in the following forms (Ewing et al 1957; Love 1967)

$$
\begin{aligned}
& \sigma_{11}=A \frac{\partial u}{\partial x}+(A-2 N) \frac{\partial v}{\partial y}+F \frac{\partial w}{\partial z}, \\
& \sigma_{22}=(A-2 N) \frac{\partial u}{\partial x}+A \frac{\partial v}{\partial y}+F \frac{\partial w}{\partial z}, \\
& \sigma_{33}=F \frac{\partial u}{\partial x}+F \frac{\partial v}{\partial y}+C \frac{\partial w}{\partial z} \\
& \sigma_{12}=N\left(\frac{\partial u}{\partial y}+\frac{\partial v}{\partial x}\right) \\
& \sigma_{23}=L\left(\frac{\partial v}{\partial z}+\frac{\partial w}{\partial y}\right) \\
& \sigma_{31}=L\left(\frac{\partial w}{\partial x}+\frac{\partial u}{\partial z}\right)
\end{aligned}
$$

where $A, N, F, C$, and $L$ are of the form given by (1). In view of the variation of the elastic parameters we have

$$
\begin{aligned}
& \sigma_{11}=e^{\nu z}\left[A_{1} \frac{\partial u}{\partial x}+\left(A_{1}-2 N_{1}\right) \frac{\partial v}{\partial y}+F_{1} \frac{\partial w}{\partial z}\right], \\
& \sigma_{22}=e^{\nu z}\left[\left(A_{1}-2 N_{1}\right) \frac{\partial u}{\partial x}+A_{1} \frac{\partial v}{\partial y}+F_{1} \frac{\partial w}{\partial z}\right], \\
& \sigma_{33}=e^{\nu z}\left[F_{1} \frac{\partial u}{\partial x}+F_{1} \frac{\partial v}{\partial y}+C_{1} \frac{\partial w}{\partial z}\right], \\
& \sigma_{12}=e^{\nu z} N_{1}\left(\frac{\partial u}{\partial y}+\frac{\partial v}{\partial x}\right) \\
& \sigma_{23}=e^{\nu z} L_{1}\left(\frac{\partial v}{\partial z}+\frac{\partial w}{\partial y}\right) \\
& \sigma_{31}=e^{\nu z} L_{1}\left(\frac{\partial w}{\partial x}+\frac{\partial u}{\partial z}\right) .
\end{aligned}
$$

Introducing (7) in (5) we get

$$
A_{1} \frac{\partial^{2} u}{\partial x^{2}}+\left(F_{1}+L_{1}\right) \frac{\partial^{2} w}{\partial x \partial z}+L_{1} \frac{\partial^{2} u}{\partial z^{2}}+\nu L_{1}\left(\frac{\partial u}{\partial z}+\frac{\partial w}{\partial x}\right)=\rho_{1} \frac{\partial^{2} u}{\partial t^{2}},
$$




$$
\begin{aligned}
& N_{1} \frac{\partial^{2} v}{\partial x^{2}}+L_{1} \frac{\partial^{2} v}{\partial z^{2}}+v L_{1} \frac{\partial v}{\partial z}=\rho_{1} \frac{\partial^{2} v}{\partial t^{2}} \\
& L_{1} \frac{\partial^{2} w}{\partial x^{2}}+\left(F_{1}+L_{1}\right) \frac{\partial^{2} u}{\partial x \partial z}+C_{1} \frac{\partial^{2} w}{\partial z^{2}}+v\left(F_{1} \frac{\partial u}{\partial z}+C_{1} \frac{\partial w}{\partial z}\right)=\rho_{1} \frac{\partial^{2} w}{\partial t^{2}}
\end{aligned}
$$

For the solution of the above equations in the case of medium $M_{1}$ we take

$$
\begin{gathered}
u(x, z, t)=P(z) e^{i \eta(x-c t)}, \\
v(x, z, t)=Q(z) e^{i \eta(x-c t)}, \\
w(x, z, t)=R(z) e^{i \eta(x-c t)},
\end{gathered}
$$

and similar set of displacements for the solution of equations for the medium $M_{2}$, the functions $P, Q, R$ being replaced by $P^{\prime}, Q^{\prime}, R^{\prime}$. This helps us to have a particular type of solutions corresponding to a group of simple harmonic waves of wave length $2 \pi / \eta$, travelling with velocity $c$. Inserting (9) in (8) we get a set of differential equations for the medium $M_{1}$ as follows:

$$
\begin{aligned}
& {\left[L_{1} D^{2}+v L_{1} D+\eta^{2}\left(\rho_{1} c^{2}-A_{1}\right)\right] P+i \eta\left[\left(F_{1}+L_{1}\right) D+v L_{1}\right] R=0,} \\
& i \eta\left[\left(F_{1}+L_{1}\right) D+v F_{1}\right] P+\left[C_{1} D^{2}+v C_{1} D+\eta^{2}\left(\rho_{1} c^{2}-L_{1}\right)\right] R=0, \\
& {\left[L_{1} D^{2}+v L_{1} D+\eta^{2}\left(\rho_{1} c^{2}-N_{1}\right)\right] Q=0,}
\end{aligned}
$$

where $D=\mathrm{d} / \mathrm{d} z, D^{2}=\mathrm{d}^{2} / \mathrm{d} z^{2}$.

For medium $M_{2}$ we get similar set of differential equations as follows:

$$
\begin{aligned}
& {\left[L_{1}^{\prime} D^{2}+v^{\prime} L_{1}^{\prime} D+\eta^{2}\left(\rho_{1}^{\prime} c^{2}-A_{1}^{\prime}\right] P^{\prime}+i \eta\left[\left(F_{1}^{\prime}+L_{1}^{\prime}\right) D+v^{\prime} L_{1}^{\prime}\right] R^{\prime}=0,\right.} \\
& i \eta\left[\left(F_{1}^{\prime}+L_{1}^{\prime}\right) D+v^{\prime} F_{1}^{\prime}\right] P^{\prime}+\left[C_{1}^{\prime} D^{2}+v^{\prime} C_{1}^{\prime} D+\eta^{2}\left(\rho_{1}^{\prime} c^{2}-L_{1}^{\prime}\right)\right] R^{\prime}=0, \\
& {\left[L_{1}^{\prime} D^{2}+v^{\prime} L_{1}^{\prime} D+\eta^{2}\left(\rho_{1}^{\prime} c^{2}-N_{1}^{\prime}\right) Q^{\prime}=0 .\right.}
\end{aligned}
$$

Equations (10) and (11) must have exponential solutions for surface waves with $u, v$, and $w$ and hence, $P, Q$, and $R$ tend to zero as $Z \rightarrow \infty$ in medium $M_{1}$. Similarly $P^{\prime}, Q^{\prime}, R^{\prime}$ tend to zero as $Z \rightarrow-\infty$ in medium $M_{2}$. Hence for medium $M_{1}$

$$
\begin{aligned}
u(x, z, t) & =\left\{K_{1} e^{-i m_{1} \eta z}+K_{2} e^{-i m_{2} \eta z}\right\} e^{i \eta(x-c t)} \\
w(x, z, t) & =\left\{\bar{K}_{1} e^{-i m_{1} \eta z}+\bar{K}_{2} e^{-i m_{2} \eta z}\right\} e^{i \eta(x-c t)}, \\
v(x, z, t) & =K_{3} e^{-i m_{3} \eta z} e^{i \eta(x-c t)} .
\end{aligned}
$$

For medium $M_{2}$,

$$
\begin{aligned}
u^{\prime}(x, z, t) & =\left\{K_{4} e^{i m_{1}^{\prime} \eta z}+K_{5} e^{i m_{2}^{\prime} \eta z}\right\} e^{i \eta(x-c t)}, \\
w^{\prime}(x, z, t) & =\left\{\bar{K}_{4} e^{i m_{1}^{\prime} \eta z}+\bar{K}_{5} e^{i m_{2}^{\prime} \eta z}\right\} e^{i \eta(x-c t)} \\
v^{\prime}(x, z, t) & =K_{6} e^{i m_{3}^{\prime} \eta z} e^{i \eta(x-c t)}
\end{aligned}
$$

where for $M_{1}, m_{j}(j=1,2)$ are the roots of

$$
m^{4}+2 i \frac{\nu}{\eta} m^{3}-\left\{L_{1}\left(\rho_{1} c^{2}-L_{1}\right)+\frac{v^{2}}{\eta^{2}} L_{1} C_{1}+C_{1}\left(\rho_{1} c^{2}-A_{1}\right)+\left(F_{1}+L_{1}\right)^{2}\right\}
$$




$$
\begin{aligned}
& \frac{1}{L_{1} C_{1}} m^{2}-i \frac{v}{\eta}\left\{L_{1}\left(\rho_{1} c^{2}-L_{1}\right)+C_{1}\left(\rho_{1} c^{2}-A_{1}\right)+\left(F_{1}+L_{1}\right)^{2}\right\} \frac{1}{L_{1} C_{1}} m \\
& \quad+\left\{\left(\rho_{1} c^{2}-L_{1}\right)\left(\rho_{1} c^{2}-A_{1}\right)+\frac{v^{2}}{\eta^{2}} L_{1} F_{1}\right\} \frac{1}{L_{1} C_{1}}=0 .
\end{aligned}
$$

For $M_{2}, m_{j}^{\prime}(j=1.2)$ are the roots of

$$
\begin{aligned}
& m^{\prime 4}+2 i \frac{\nu^{\prime}}{\eta} m^{\prime 3}-\left\{L_{1}^{\prime}\left(\rho_{1}^{\prime} c^{2}-L_{1}^{\prime}\right)+\frac{\nu^{\prime 2}}{\eta^{2}} L_{1}^{\prime} C_{1}^{\prime}+C_{1}^{\prime}\left(\rho_{1}^{\prime} c^{2}-A_{1}^{\prime}\right)+\left(F_{1}^{\prime}+L_{1}^{\prime}\right)^{2}\right\} \\
& \frac{1}{L_{1}^{\prime} C_{1}^{\prime}} m^{\prime 2}-i \frac{\nu^{\prime}}{\eta}\left\{L_{1}^{\prime}\left(\rho_{1}^{\prime} c^{2}-L_{1}^{\prime}\right)+C_{1}^{\prime}\left(\rho_{1}^{\prime} c^{2}-A_{1}^{\prime}\right)+\left(F_{1}^{\prime}+L_{1}^{\prime}\right)^{2}\right\} \frac{1}{L_{1}^{\prime} C_{1}^{\prime}} m^{\prime} \\
& \quad+\left\{\left(\rho_{1}^{\prime} c^{2}-L_{1}^{\prime}\right)\left(\rho_{1}^{\prime} c^{2}-A_{1}^{\prime}\right)+\frac{\nu^{\prime 2}}{\eta^{2}} L_{1}^{\prime} F_{1}^{\prime}\right\} \frac{1}{L_{1}^{\prime} C_{1}^{\prime}}=0,
\end{aligned}
$$

with positive real part and

$$
\begin{array}{ll}
\bar{K}_{1}=\alpha_{1} K_{1}, & \bar{K}_{2}=\alpha_{2} K_{2}, \\
\bar{K}_{4}=\alpha_{1}^{\prime} K_{4}, & \bar{K}_{5}=\alpha_{2}^{\prime} K_{5},
\end{array}
$$

where

$$
\alpha_{j}=\frac{L_{1} m_{j}^{2}+i \frac{\nu}{\eta} L_{1} m_{j}-\left(\rho_{1} c^{2}-A_{1}\right)}{\left(F_{1}+L_{1}\right) m_{j}+i \frac{v}{\eta} L_{1}}
$$

and

$$
\alpha_{j}^{\prime}=\frac{L_{1}^{\prime} m_{j}^{\prime 2}+i \frac{v^{\prime}}{\eta} L_{1}^{\prime} m_{j}^{\prime}-\left(\rho_{1}^{\prime} C^{2}-A_{1}^{\prime}\right)}{i \frac{v^{\prime}}{\eta} L_{1}^{\prime}-\left(F_{1}^{\prime}+L_{1}^{\prime}\right) m_{j}^{\prime}}, \quad j=1,2 .
$$

Also $m_{3}$ is a root of

$$
L_{1} m^{2}+i \frac{v}{\eta} L_{1} m-\left(\rho_{1} c^{2}-N_{1}\right)=0
$$

and $m_{3}^{\prime}$ is a root of

$$
L_{1}^{\prime} m^{\prime 2}+i \frac{\nu^{\prime}}{\eta} L_{1}^{\prime} m^{\prime}-\left(\rho_{1}^{\prime} c^{2}-N_{1}^{\prime}\right)=0 .
$$

\section{Boundary conditions}

(i) The displacement components at the interface between $M_{1}$ and $M_{2}$ must be continuous.

(ii) The stresses $\sigma_{31}, \sigma_{32}, \sigma_{33}$ are also continuous at the interface, where

$$
\begin{aligned}
& \sigma_{31}=e^{\nu z} L_{1}\left(\frac{\partial u}{\partial z}+\frac{\partial w}{\partial x}\right), \\
& \sigma_{32}=e^{\nu z} L_{1} \frac{\partial v}{\partial z} \\
& \sigma_{33}=e^{\nu z}\left(F_{1} \frac{\partial u}{\partial x}+C_{1} \frac{\partial w}{\partial z}\right) .
\end{aligned}
$$




\section{Solutions of the problem}

Applying the above boundary conditions we have

$$
\begin{aligned}
& K_{1}+K_{2}-K_{4}-K_{5}=0 \\
& \alpha_{1} K_{1}+\alpha_{2} K_{2}-\alpha_{1}^{\prime} K_{4}-\alpha_{2}^{\prime} K_{5}=0 \\
& K_{3}=K_{6} \\
& L_{1}\left(m_{1}-\alpha_{1}\right) K_{1}+L_{1}\left(m_{2}-\alpha_{2}\right) K_{2}+L_{1}^{\prime}\left(m_{1}^{\prime}+\alpha_{1}^{\prime}\right) K_{4}+L_{1}^{\prime}\left(m_{2}^{\prime}+\alpha_{2}^{\prime}\right) K_{5}=0 \\
& \left(C_{1} m_{1} \alpha_{1}-F_{1}\right) K_{1}+\left(C_{1} m_{2} \alpha_{2}-F_{1}\right) K_{2}+\left(C_{1}^{\prime} m_{1}^{\prime} \alpha_{1}^{\prime}+F_{1}^{\prime}\right) K_{4} \\
& \quad+\left(C_{1}^{\prime} m_{2}^{\prime} \alpha_{2}^{\prime}+F_{1}^{\prime}\right) K_{5}=0 \\
& L_{1} m_{3} K_{3}+L_{1}^{\prime} m_{3}^{\prime} K_{6}=0 .
\end{aligned}
$$

The third and the sixth conditions in (20) imply that there is no transverse component of displacement.

Eliminating constants $K_{1}, K_{2}, K_{4}, K_{5}$ from (20) we get

$$
\operatorname{det}\left(a_{i j}\right)=0, \quad i, j=1,2,3,4 \text {, }
$$

where

$$
\begin{aligned}
& a_{11}=1, \quad a_{12}=1, \quad a_{13}=-1, \quad a_{14}=-1 \\
& a_{21}=\alpha_{1}, \quad a_{22}=\alpha_{2}, \quad a_{23}=-\alpha_{1}^{\prime}, \quad a_{24}=-\alpha_{2}^{\prime} \\
& a_{31}=L_{1}\left(m_{1}-\alpha_{1}\right), \quad a_{32}=L_{1}\left(m_{2}-\alpha_{2}\right), \\
& a_{33}=L_{1}^{\prime}\left(m_{1}^{\prime}+\alpha_{1}^{\prime}\right), \quad a_{34}=L_{1}^{\prime}\left(m_{2}^{\prime}+\alpha_{2}^{\prime}\right) \\
& a_{41}=c_{1} m_{1} \alpha_{1}-F_{1}, \quad a_{42}=c_{1} m_{2} \alpha_{2}-F_{1}, \\
& a_{43}=C_{1}^{\prime} m_{1}^{\prime} \alpha_{1}^{\prime}+F_{1}^{\prime}, \quad a_{44}=C_{1}^{\prime} m_{2}^{\prime} \alpha_{2}^{\prime}+F_{1}^{\prime} .
\end{aligned}
$$

The roots of (21) determine the wave velocity of the generalized interface wave propagating along the common boundary of two inhomogeneous transversely isotropic elastic solid media. If we take $v=v^{\prime}=0$ i.e., a homogeneous medium, we obtain the equation obtained by Dey \& Sengupta (1978), where in $M_{1}$

$$
\begin{aligned}
& m_{1}^{2}+m_{2}^{2}=\left\{L_{1}\left(\rho_{1} c^{2}-L_{1}\right)+C_{1}\left(\rho_{1} c^{2}-A_{1}\right)+\left(F_{1}+L_{1}\right)^{2}\right\} \frac{1}{L_{1} C_{1}} \\
& m_{1}^{2} \cdot m_{2}^{2}=\left(\rho_{1} c^{2}-A_{1}\right)\left(\rho_{1} c^{2}-L_{1}\right) \frac{1}{L_{1} C_{1}} \\
& \alpha_{1}=\frac{L_{1} m_{1}^{2}-\left(\rho_{1} c^{2}-A_{1}\right)}{\left(F_{1}+L_{1}\right) m_{1}} \\
& \alpha_{2}=\frac{L_{1} m_{2}^{2}-\left(\rho_{1} c^{2}-A_{1}\right)}{\left(F_{1}+L_{1}\right) m_{2}}
\end{aligned}
$$

and similar expressions in $M_{2}$. 


\section{Rayleigh waves}

For Rayleigh waves, $M_{2}$ is replaced by vacuum. Hence from (20)

$$
\begin{aligned}
& \left(m_{1}-\alpha_{1}\right) K_{1}+\left(m_{2}-\alpha_{2}\right) K_{2}=0, \\
& \left(C_{1} m_{1} \alpha_{1}-F_{1}\right) K_{1}+\left(C_{1} m_{2} \alpha_{2}-F_{1}\right) K_{2}=0 .
\end{aligned}
$$

Eliminating $K_{1}, K_{2}$ from (22) we get

$$
\left(\alpha_{2}-\alpha_{1}\right)\left(C_{1} m_{1} m_{2}-F_{1}\right)+\left(m_{1}-m_{2}\right)\left(C_{1} \alpha_{1} \alpha_{2}-F_{1}\right)=0 .
$$

Equation (23) gives the wave velocity for Rayleigh waves in a transversely isotropic nonhomogeneous medium, the nonhomogeneity being of the assumed forms (1), (2). It is evident from (23) that the values of $m_{1}, m_{2}$ depend on the particular value of $\eta$ and hence the wave velocity $c$ depends on $\eta$ creating a dispersion of the general wave form.

Equation (23) is of the same form as obtained by Dey \& Sengupta (1978) except that $m_{1}, m_{2}$ are different. Now $m_{1}, m_{2}$ depend upon $v$ as evident from (14) whereas for homogeneous media they are obtained from

$$
\begin{aligned}
& m^{4}-\left\{L_{1}\left(\rho_{1} c^{2}-L_{1}\right)+C_{1}\left(\rho_{1} c^{2}-A_{1}\right)+\left(F_{1}+L_{1}\right)^{2}\right\} \frac{1}{L_{1} C_{1}} m^{2} \\
& \quad+\left\{\left(\rho_{1} c^{2}-A_{1}\right)\left(\rho_{1} c^{2}-L_{1}\right)\right\} \frac{1}{L_{1} C_{1}}=0 .
\end{aligned}
$$

\section{Love waves}

We take the origin of the coordinate system at the interface between the upper stratum of thickness $H(-H \leq Z \leq 0)$ and the lower semi-infinite medium $(0 \leq Z<\infty)$ with $x$-axis and $z$-axis taken along the interface and vertically downwards respectively (figure 2 ).

For Love wave propagation along the $x$-axis we have $u=0, w=0, v=v(x, z, t)$. Therefore, the only equation of motion for medium $M_{1}$ is

$$
N_{1} \frac{\partial^{2} v_{1}}{\partial x^{2}}+v L_{1} \frac{\partial v_{1}}{\partial z}+L_{1} \frac{\partial^{2} v_{1}}{\partial z^{2}}=\rho_{1} \frac{\partial^{2} v_{1}}{\partial t^{2}}
$$



Figure 2. Love waves. 
and for medium $M_{2}$

$$
N_{1}^{\prime} \frac{\partial^{2} v_{2}}{\partial x^{2}}+v^{\prime} L_{1}^{\prime} \frac{\partial v_{2}}{\partial z}+L_{1}^{\prime} \frac{\partial^{2} v_{2}}{\partial z^{2}}=\rho_{1}^{\prime} \frac{\partial^{2} v_{2}}{\partial t^{2}}
$$

Putting $v_{j}=V_{j}(x, z) e^{i \lambda t}(j=1,2$ and $\lambda$ is the wave frequency) (25) and (26) reduce to

$$
\begin{aligned}
& N_{1} \frac{\partial^{2} V_{1}}{\partial x^{2}}+v L_{1} \frac{\partial V_{1}}{\partial z}+L_{1} \frac{\partial^{2} V_{1}}{\partial z^{2}}+\rho_{1} \lambda^{2} V_{1}=0 \\
& N_{1}^{\prime} \frac{\partial^{2} V_{2}}{\partial x^{2}}+v^{\prime} L_{1}^{\prime} \frac{\partial V_{2}}{\partial z}+L_{1}^{\prime} \frac{\partial^{2} V_{2}}{\partial z^{2}}+\rho_{1}^{\prime} \lambda^{2} V_{2}=0
\end{aligned}
$$

Let $\bar{f}(K, Z)$ be the Fourier transform of $f(x, z)$ defined by the relation

$$
\bar{f}(K, Z)=\int_{-\infty}^{\infty} f(x, z) e^{i k x} \mathrm{~d} x,
$$

so that

$$
f(x, z)=\frac{1}{2 \pi} \int_{-\infty}^{\infty} \bar{f}(K, Z) e^{-i k z} \mathrm{~d} k .
$$

Applying Fourier transformation defined by (29) we obtain equations of motion (27) and (28) in the form

$$
\begin{aligned}
& \frac{\mathrm{d}^{2} \bar{V}_{1}}{\mathrm{~d} z^{2}}+v \frac{\mathrm{d} \bar{V}_{1}}{\mathrm{~d} z}+P_{1}^{2} \bar{V}_{1}=0 \\
& \frac{\mathrm{d}^{2} \bar{V}_{2}}{\mathrm{~d} z^{2}}+v^{\prime} \frac{\mathrm{d} \bar{V}_{2}}{\mathrm{~d} z}+P_{2}^{2} \bar{V}_{2}=0
\end{aligned}
$$

where

$$
\begin{aligned}
& P_{1}^{2}=\frac{\rho_{1}}{L_{1}} \lambda^{2}-\frac{N_{1}}{L_{1}} K^{2}, \\
& P_{2}^{2}=\frac{\rho_{1}^{\prime}}{L_{1}^{\prime}} \lambda^{2}-\frac{N_{1}^{\prime}}{L_{1}^{\prime}} K^{2} .
\end{aligned}
$$

We take solutions of (30) and (31) as follows.

$$
\begin{aligned}
& \bar{V}_{1}=S e^{-\gamma_{1} z}, \\
& \bar{V}_{2}=\gamma e^{-\gamma_{2} z}+T e^{\gamma_{3} z},
\end{aligned}
$$

where

$$
\begin{aligned}
& \gamma_{1}=\frac{v}{2}-i\left(\frac{\lambda^{2}}{\beta_{1}^{2}}-\frac{N_{1}}{L_{1}} K^{2}-\frac{\nu^{2}}{4}\right)^{1 / 2}, \\
& \gamma_{2}=\frac{\nu^{\prime}}{2}-i\left(\frac{\lambda^{2}}{\beta_{2}^{2}}-\frac{N_{1}^{\prime}}{L_{1}^{\prime}} K^{2}-\frac{\nu^{\prime} 2}{4}\right)^{1 / 2}, \\
& \gamma_{3}=-\frac{v^{\prime}}{2}-i\left(\frac{\lambda^{2}}{\beta_{2}^{2}}-\frac{N_{1}^{\prime}}{L_{1}^{\prime}} K^{2}-\frac{v^{\prime 2}}{4}\right)^{1 / 2}, \\
& \beta_{1}^{2}=\frac{L_{1}}{\rho_{1}}, \quad \beta_{2}^{2}=\frac{L_{1}^{\prime}}{\rho_{1}^{\prime}} .
\end{aligned}
$$


Thus the displacements in these two media are

$$
V_{1}=\frac{1}{2 \pi} \int_{-\infty}^{\infty} S e^{-\gamma_{1} z} e^{-i k x} \mathrm{~d} k
$$

and

$$
V_{2}=\frac{1}{2 \pi} \int_{-\infty}^{\infty}\left(Y e^{-\gamma_{2} z}+T e^{\gamma_{3} z}\right) e^{-i k x} \mathrm{~d} k
$$

Applying the boundary conditions

(i) $\quad V_{1}=V_{2}$

(ii) $\left.L_{1} \frac{\partial V_{1}}{\partial z}=L_{1}^{\prime} \frac{\partial V_{2}}{\partial z}\right\}$ on $z=0$,

and

$$
\text { (iii) } \quad L_{1}^{\prime} \frac{\partial V_{2}}{\partial z}=0 \text {, on } Z=-H
$$

we get

$$
\begin{aligned}
& S-Y-T=0, \\
& L_{1} \gamma_{1} S-L_{1}^{\prime} \gamma_{2} Y+L_{1}^{\prime} \gamma_{3} T=0, \\
& \gamma_{2} e^{\gamma_{2} H} Y-\gamma_{3} e^{-\gamma_{3} H} T=0 .
\end{aligned}
$$

Eliminating $S, Y, T$ from the set of equations (38) and taking $\lambda=c K$, where $c$ is the common wave velocity propagating along the $x$-axis we obtain the equation of dispersion as

$$
\begin{aligned}
\tan & \left\{K H\left(\frac{c^{2}}{\beta_{2}^{2}}-\frac{N_{1}^{\prime}}{L_{1}^{\prime}}-\frac{v^{\prime} 2}{4 K^{2}}\right)^{1 / 2}\right\} \\
= & \left\{\frac{L_{1}}{L_{1}^{\prime}}\left[\frac{c^{2}}{\beta_{2}^{2}}-\frac{N_{1}^{\prime}}{L_{1}^{\prime}}-\frac{v^{\prime 2}}{4 K^{2}}\right]^{1 / 2}\left(\frac{v}{2 K}+\left[\frac{N_{1}}{L_{1}}+\frac{v^{2}}{4 K^{2}}-\frac{c^{2}}{\beta_{1}^{2}}\right]^{1 / 2}\right)\right\} \div \\
& \left\{\frac{c^{2}}{\beta_{2}^{2}}-\frac{N_{1}^{\prime}}{L_{1}^{\prime}}-\frac{L_{1}}{L_{1}^{\prime}} \cdot \frac{v^{\prime}}{2 K}\left(\frac{v}{2 K}+\left[\frac{N_{1}}{L_{1}}+\frac{v^{2}}{4 K^{2}}-\frac{c^{2}}{\beta_{1}^{2}}\right]^{1 / 2}\right)\right\} .
\end{aligned}
$$

For the real solution of (39) we note that

$$
\left(\frac{N_{1}^{\prime}}{L_{1}^{\prime}}+\frac{v^{\prime 2}}{4 K^{2}}\right)^{1 / 2}<\frac{c}{\beta_{2}}<\frac{\beta_{1}}{\beta_{2}}\left(\frac{N_{1}}{L_{1}}+\frac{v^{2}}{4 K^{2}}\right)^{1 / 2}
$$

If the nonhomogeneity of the material media be neglected, putting $v=v^{\prime}=0$ in the above dispersion equation, we obtain the wave velocity equation for Love waves in a transversely isotropic medium as

$$
\begin{aligned}
& \tan K H\left[\frac{c^{2}}{\beta_{2}^{2}}-\frac{N_{1}^{\prime}}{L_{1}^{\prime}}\right]^{1 / 2} \\
& \quad=\frac{L_{1}}{L_{1}^{\prime}}\left\{\left[\frac{c^{2}}{\beta_{2}^{2}}-\frac{N_{1}^{\prime}}{L_{1}^{\prime}}\right]^{1 / 2}\left[\frac{N_{1}}{L_{1}}-\frac{c^{2}}{\beta_{1}^{2}}\right]^{1 / 2}\right\} /\left\{\frac{N_{1}^{\prime}}{L_{1}^{\prime}}-\frac{c^{2}}{\beta_{2}^{2}}\right\}
\end{aligned}
$$




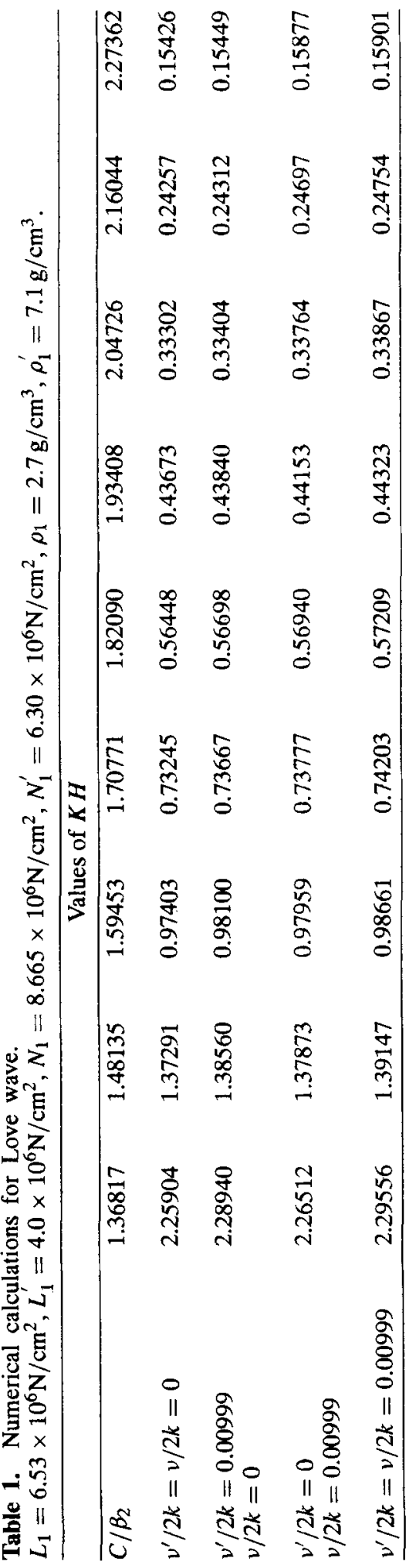


where

$$
\left(\frac{N_{1}^{\prime}}{L_{1}^{\prime}}\right)^{1 / 2}<\frac{c}{\beta_{2}}<\frac{\beta_{1}}{\beta_{2}}\left(\frac{N_{1}}{L_{1}}\right)^{1 / 2}
$$

From the above it is observed that the inhomogeneity of the material media has a distinct effect on the range of admissible values of the phase velocity of Love waves.

\section{Stoneley waves}

The generalized form of Rayleigh waves which propagate along the interface of $M_{1}$ and $M_{2}$ are known as Stoneley waves according to the classical theory. Hence, in the present case of anisotropic inhomogeneous elastic media, Stoneley waves propagating along the common boundary of $M_{1}$ and $M_{2}$ are determined by the roots of the wave velocity equation (21). It is seen from this equation that Stoneley wave velocity equation is influenced by the nonhomogeneous and anisotropic nature of the material media. Putting $v=v^{\prime}=0$ in (21) one obtains the wave velocity equation for Stoneley waves in transversely isotropic homogeneous elastic media.

\section{Discussion}

Table 1 shows that the inhomogeneity has an important role to play on Love wave propagation in transversely isotropic elastic media. The table also indicates that the phase velocity decreases with increase of $K H$, i.e. the thickness of the layer. Moreover, for a particular value of wave velocity, the values of $K H$ increase with increase of the inhomogeneous character of material media.

The authors take this opportunity to express their gratitude to the reviewer of this paper for his/her valuable comments and suggestions towards its improvement.

\section{References}

Bhattacharya J 1969 The possibility of the propagation of Love type waves in an intermediate heterogeneous layer lying between two semi-infinite isotropic homogeneous elastic layers. Pure Appl. Geophys. 72: 61-67

Biot M A 1965 Mechanics of incremental deformations (New York: John Wiley and Sons)

Bland D R 1960 The theory of linear visco-elasticity (London: Pergamon) (This monograph on the subject contains many cases of stress analysis)

Chattopadhay A 1975 On the propagation of Love type waves in an intermediate nonhomogeneous layer lying between two semi-infinite homogeneous elastic media. Gerlands Beitr: Geophys. 84: 327-334

Dutta S 1963a Love waves in a non-homogeneous internal stratum lying between two semi-infinite isotropic media. Geophysics 28: 156-160 
Dutta S 1963 b On the propagation of Love waves in a non-homogeneous internal stratum of finite depth lying between two semi-infinite isotropic media. Geophys. Pure Appl. 55: 31-36

Dey S K, Sengupta P R 1978 Effects of anisotropy on surface waves under the influence of gravity. Acta Geophys. Polon. 26: 291-298

Das S C, Acharya D P, SenGupta P R 1992 Surface waves in an inhomogeneous elastic medium under the influence of gravity. Revue. Roumaine Des Sc. Techn. Mec. Appl. 37: 539-552

Ewing W M, Jardetzky W S, Press F 1957 Elastic waves in layered media. (New York-TorontoLondon: McGraw-Hill) pp 348-350

Flugge W 1967 Visco-elasticity (London: Blaisdeli)

Hunter S C 1960 Visco-elastic waves. Progress in solid mechanics (eds) I N Snedon, R Hill (Amsterdam/New York: North-Holland/Interscience)

Jeffreys H 1970 The Earth (Cambridge: University Press)

Love A E H 1967 A treatise on the mathematical theory of elasticity (New York: Dover)

Rayleigh Lord 1885 On wave propagation along the plane surface of an elastic solid. Proc. London Math. Soc. 17: 4-11

Stoneley R 1924 The elastic waves at the surface of separation of two solids. Proc. R. Soc. London A106: $416-428$ 